\title{
A prospective study of work-private life conflict and number of pain sites: moderated mediation by sleep problems and support
}

\author{
Jolien Vleeshouwers $^{1}$ (D) $\cdot$ Stein Knardahl $^{1} \cdot$ Jan Olav Christensen $^{1}$
}

Received: May 10, 2017 / Accepted: July 20, 2018/Published online: July 25, 2018

(C) The Author(s) 2018

\begin{abstract}
The objective of the current study was to elucidate how work-private life conflict prospectively affects musculoskeletal pain complaints by exploring possible mediation through sleep problems. In addition, the study determined whether support from coworkers and superior moderate this mediated relationship. The study incorporated a two-wave full panel design and participants included 4681 Norwegian working men and women. Path analyses were performed to study direct and indirect effects of work-private life conflict on sleep problems and multisite musculoskeletal pain, moderated by support. This study suggested time-lagged relationships of work-private life conflict with number of pain sites. Furthermore, sleep problems may mediate the effects of work-private life conflict on number of pain sites. While support has been found to affect the direct relationship between work-private life conflict and number of pain sites, it does not significantly moderate the indirect mediation effect, i.e. no moderated mediation effect of support was established. Findings from the present study suggest sleep may be one explaining factor in the complex work-pain mechanism, and this may aid the development of theories on workprivate life conflict and pain. Since both work-private life conflict and support are modifiable work factors, primary workplace interventions by the employer aiming to reduce sleep problems and musculoskeletal pain in employees
\end{abstract}

Electronic supplementary material The online version of this article (https://doi.org/10.1007/s10865-018-9957-0) contains supplementary material, which is available to authorized users.

Jolien Vleeshouwers

jolien.vleeshouwers@stami.no

1 Department of Work Psychology and Physiology, The National Institute of Occupational Health, Oslo, Norway could target these specific work factors, and help prevent work-related pain complaints.

Keywords Work-private life conflict - Sleep · Multisite pain $\cdot$ Mediation $\cdot$ Moderation $\cdot$ Support

\section{Introduction}

Musculoskeletal pain is among the most frequently reported health complaints in the working population and is a common cause of long-term illness and sick leave (Woolf \& Pfleger, 2003). The relationship between psychosocial work factors and single anatomical pain sites (particularly neck and back) has been studied extensively (Christensen \& Knardahl, 2010, 2012). However, more recently the study of co-occurring pain sites has gained attention (Neupane et al., 2013). Multisite musculoskeletal pain, i.e. reporting more than one concurrent pain site, is more prevalent than single-site pain (Kamaleri et al., 2008b) and may have a greater impact on health (Neupane et al., 2013), sick leave (Haukka et al., 2013), and work disability (Miranda et al., 2010). While some psychological work factors are established predictors of pain complaints, including multisite pain (Christensen \& Knardahl, 2010, 2012; Solidaki et al., 2010), many potential predictors remain less studied. Moreover, the processes by which work factors affect pain complaints are unknown, calling for studies that identify factors on the pathway from work to health.

Multisite pain can be defined as 'pain in more than one body location concurrently or alternatively within a defined time period' (Paananen, 2011). Some have argued pain should occur in at least one upper limb and one lower limb as well as both on the left as well as the right side of the body (Wolfe et al., 2010), i.e. pain should be widespread to 
be considered multisite pain (Dean et al., 2017). However, others have argued that multisite pain should simply be based on the number of body sites affected, regardless of their anatomical distribution (Coggon et al., 2013). Kamaleri et al. (2009) observed a strong prospective, long term dose-response relationship between the number of pain sites and work disability concluding that counting the number of pain sites is a simple and powerful way to assess risk.

\section{Work-private life conflict affecting sleep and pain}

For many employees, coping with demands both at work and at home is challenging. Two roles requiring time, attention, and effort may result in conflict between workand private life. With the emergence of 'New ways of working (NWW)', which is characterized by flexibility in work hours, but also by increased accessibility via smartphone and email, (Demerouti et al., 2014) this concern may seem more pressing than ever. Work-private life conflict may result in negative health effects such as distress, sleep deprivation, and musculoskeletal pains (Christensen \& Knardahl, 2010, 2012; Hammig \& Bauer, 2014; Hammig et al., 2009; Kim et al., 2013). Contemporary families encompass many configurations and private life responsibilities may extend beyond family obligations. Therefore, the present study addresses work-private life conflict (WLC) rather than work-family conflict alone.

Mechanisms have been proposed for how psychological and social factors at work may affect both sleep and pain (Davis \& Heaney, 2000; Hoogendoorn et al., 2000). A conflict between demands from work and private life occurs because these separate demands are directed towards the same limited resources, therefore resulting in loss at either end, e.g. valued roles at home or at work, or time spent at either place (Crain et al., 2014). In the instance of WLC leading to sleep problems and pain, one possible way in which sleep may mediate the relationship is if challenges at work influence the employee's sparetime and home situation. In order to compensate lost time at home, the employee may spend additional time investing in their 'home role', likely cutting into sleep time (Barnes et al., 2012; Crain et al., 2014). In addition, worries about work may disrupt sleep.

Work-private life conflict has been linked to musculoskeletal pain (Hammig \& Bauer, 2014; Hammig et al., 2009; Kim et al., 2013) as well as sleeping difficulties (Crain et al., 2014; Hammig et al., 2009; Lallukka et al., 2010; Vleeshouwers et al., 2016). While studied to a lesser degree, WLC has been linked to multisite pain specifically as well (Aili et al., 2015). However, WLC has to our knowledge not been studied together with sleep, support, and NPS simultaneously.
A population-representative Swiss cross-sectional study revealed associations between higher levels of WLC and poorer health, including back- and neck pain, and sleep problems (Hammig et al., 2009). Other studies have also suggested both WLC and support may affect sleep (Hammig et al., 2009; Jacobsen et al., 2014; Magnusson Hanson et al., 2014; Nakata et al., 2004). One longitudinal study of the relationship between WLC and sleep (Jacobsen et al., 2014) found that WLC predicted sleep deficiency nearly 2 years later.

\section{Sleep affecting pain}

The relationship between sleep and pain has long been acknowledged (Matre et al., 2015; Moldofsky, 2001; Sivertsen et al., 2015). Pain may interfere with sleep patterns and behaviors, and sleep disruption has been found to influence pain (Alsaadi et al., 2014; Moldofsky, 2001). Furthermore, sleep restriction has been found to elevate pain sensitivity (Haack et al., 2007; Matre et al., 2015; Sivertsen et al., 2015).

Knowing that sleep quality may be influenced by work factors (Vleeshouwers et al., 2016) and that sleep may influence pain, including multisite pain specifically (Aili et al., 2015), one may surmise that sleep problems could be one of several factors responsible for the link between work and pain.

\section{Support as a buffer}

Receiving support at work from either colleagues or supervisors may have beneficial health effects (Bongers et al., 1993; Eriksen et al., 2004). Low levels of support from one's superior at work may predict difficulties initiating and maintaining sleep (Vleeshouwers et al., 2016), while low support from family and coworkers be associated with difficulties initiating and maintaining sleep (Nakata et al., 2004). Support has also been associated with musculoskeletal pain complaints (Aili et al., 2015; Bongers et al., 1993; Eriksen et al., 2004; Solidaki et al., 2010). Moreover, support may affect WLC (Kossek et al., 2011). In terms of COR theory, support may act as a resource when other resources, such as time, are depleted due to work-private life conflict (Hobfoll et al., 1990). Work-related social support and understanding from the workplace regarding demands at home may affect the consequences of work- and private life on health outcomes. Work-related support has been studied as a moderator in relation to WLC and other work factors (Ganster et al., 1986). For example, support has been found to moderate the relationships between WLC and job satisfaction and between WLC and organizational commitment (Ganster et al., 1986). However, it seems the role of work-related social support as a 
potential buffering mechanism between work and health outcomes, including sleep, is underexplored and unresolved, therefore needing further investigation.

\section{Hypotheses}

Most studies on WLC and pain have been cross-sectional and have focused on specific work populations, e.g. women, hospital personnel, or white-collar workers (Berntsson et al., 2006; Hammig \& Bauer, 2014; Kim et al., 2013). Therefore, the mechanisms by which WLC influences health and pain remain underexplored. A more in-depth evaluation of this specific psychosocial work factor, and the addition of mediating and moderating factors such as sleep and support to these models, should add to the understanding of how work may affect employee health. Moreover, exploring effects of WLC on subsequent sleep problems and NPS particularly relevant in practice as WLC, sleep, and support are all modifiable factors that represent highly relevant targets of interventions to improve health and well-being.

Thus, the current study tested the hypotheses that workprivate life conflict (WLC) predicts the number of pain sites (NPS) employees reported 2 years after (Hypothesis 1: H1), that sleep problems predict NPS 2 years after $(\mathrm{H} 2)$, and that sleep problems mediate the relationship of WLC with subsequent NPS (H3). That is, WLC may affect sleep, which in turn may affect pain, thereby suggesting one possible mechanism to account for the putative health impact of WLC. Furthermore, we tested whether workrelated social support from coworkers and superiors to attenuate this relationship, i.e. buffer the impact of WLC on NPS (H4).

\section{Methods}

\section{Design}

The present two-wave prospective full-panel study included parts of a large questionnaire distributed by the Norwegian National Institute of Occupational Health. This questionnaire gathered data on background information, work organization, psychological and social work factors, coping strategies, attitudes towards work, personality, mental health, and physical health complaints. Data were collected from November 2004 until November 2012. For each participant follow-up data was collected 2 years after baseline.

\section{Participants}

While many previous studies have mainly included working men and women with children living at home, effec- tively focusing on work-family life conflict, the present study includes all working men and women, including single parents and dual-income couples without children. Participants were recruited at the organizational level from participating companies, covering both private- and public sector workplaces, and including for example insurance companies, local government, and healthcare institutions. After receiving a letter with a unique code, participants could choose to complete the online questionnaire or the paper version. Participating organizations were obligated to allow their employees to complete the survey during working hours; however, participants could also choose to complete the questionnaire at home.

For the present study, 12,656 participants from 63 different companies were invited to partake and complete the questionnaire at both baseline and follow-up. Hereof, 4789 (37.8\%) employees completed all relevant items at baseline and $4716(37.2 \%)$ employees also completed all items at follow-up. After exclusion due to missing information about sex, age, and skill level, the final sample size was $4681(37.0 \%$ of all invited, $97.7 \%$ of those that completed the form at baseline). Of these participants, $44.9 \%$ were male and $55.1 \%$ were female (see Table 1 ).

\section{Outcome measures: number of pain sites}

NPS was measured by summing up the number of selfreported musculoskeletal pain sites (Kamaleri et al., 2008a). Simple numerical pain measures are found to be more successful in accurately classifying and understanding pain in the general public then more advanced measures (Schmidt \& Baumeister, 2007). Musculoskeletal pain items reflected occurrence of 'being troubled by' pain in the specific body sites. In the Norwegian language 'being troubled by' is a common way of describing discomfort through pain. Response alternatives included: "not troubled", "a little troubled", "somewhat troubled", and "intensely troubled".

Five separate musculoskeletal pain sites were measured; (1) neck pain, (2) pain in the shoulder and/or upper arm, (3) pain in the underarm and/or hands, (4) back pain, and (5) pain in the legs. Scores on these items were dichotomized, with the category for being "a little troubled" in the last 4 weeks by pain in that particular pain site functioning as the cut-off point. The sum of the number of pain items reported was calculated to reflect the NPS variable.

\section{Exposure measure; work-private life conflict (WLC)}

WLC was measured by items from the General Nordic Questionnaire for Psychological and Social Factors at Work (QPSNordic) (Lindström, 2000). WLC was quanti- 
Table 1 Baseline descriptives for the study sample $(\mathrm{N}=4681)$

\begin{tabular}{|c|c|c|}
\hline & $\mathrm{N} /$ mean & $\%$ \\
\hline Age & Mean: 44.6, sd: 10.2 & - \\
\hline \multicolumn{3}{|l|}{ Sex } \\
\hline Male & 2168 & 46.3 \\
\hline Female & 2513 & 53.7 \\
\hline \multicolumn{3}{|l|}{ Skill level } \\
\hline$>16$ years & 1398 & 29.9 \\
\hline $13-16$ years & 863 & 18.4 \\
\hline $10-12$ years & 1849 & 39.5 \\
\hline$<10$ years & 29 & 0.6 \\
\hline Unspecified & 542 & 11.6 \\
\hline \multicolumn{3}{|c|}{ Work-private life conflict } \\
\hline Range $1-5$ & Mean: 1.96 , sd: 0.75 & \\
\hline \multicolumn{3}{|l|}{ Social support } \\
\hline Range $1-5$ & Mean: 4.01, sd: 0.77 & \\
\hline \multicolumn{3}{|l|}{ NPS T1 } \\
\hline 0 & 1053 & 22.5 \\
\hline 1 & 1053 & 22.5 \\
\hline 2 & 1005 & 21.5 \\
\hline 3 & 838 & 17.9 \\
\hline 4 & 507 & 10.8 \\
\hline 5 & 225 & 4.8 \\
\hline Mean count & 1.86 & - \\
\hline \multicolumn{3}{|l|}{ NPS T2 } \\
\hline 0 & 1023 & 21.9 \\
\hline 1 & 1028 & 22.0 \\
\hline 2 & 1065 & 22.8 \\
\hline 3 & 777 & 16.6 \\
\hline 4 & 531 & 11.3 \\
\hline 5 & 257 & 5.5 \\
\hline Mean count & 1.90 & - \\
\hline \multicolumn{3}{|l|}{ Pain prevalence } \\
\hline Neck pain & 2255 & 48.2 \\
\hline Shoulder pain & 2016 & 43.1 \\
\hline Arm pain & 1114 & 23.8 \\
\hline Back pain & 2085 & 44.5 \\
\hline Leg pain & 1260 & 26.9 \\
\hline
\end{tabular}

fied as the interplay between work and private life and consisted of two items measured on a 5-point scale. The first item reflects how often the participant feels work interferes with their private- or family life ("Do you feel that demands from the workplace interfere with your private- and family life?"), while the second item reflects the degree to which the participant feels their private life interferes with their work ("Do you feel that demands from your private- and family life interfere with how you execute your work"). WLC scores were calculated by com- puting the mean score of the items. Baseline correlation for WLC items was $\rho 0.61, p<.01$.

\section{Potential mediator: sleep problems}

Sleep problems was measured with two items, namely difficulty initiating sleep and disturbed sleep (Harvey et al., 2008). Items were measured with the following question: "Have you experienced the following symptoms in the last 4 weeks?" followed by the two items. Response alternatives were: " 0 times", " $1-3$ times per month", " $1-2$ times per week", "3-5 times per week", and "6-7 times per week". Sleep problems scores were calculated by computing the sum score of items. Baseline correlation for the two sleep items was $\rho 0.81, p<.01$.

\section{Potential moderator; support}

Work-related social support was measured with five Likerttype items from the QPSNordic (Lindström, 2000); two reflecting the first subscale of co-worker support ("When needed, can you ask colleagues for help or support with your work?" and "When needed, are colleagues willing to listen to- and discuss problems you experience in your work?") and three reflecting the second subscale of supervisor support ("When needed, can you ask your closest supervisor for help or support with your work?", "When needed, is your closest supervisor willing to listen to- and discuss problems you experience in your work?", and "Are your work achievements appreciated by your closest supervisor?"). Response categories reflected frequency of occurrence and ranged from " $1=$ very seldom to never" to " $5=$ very often or always". Support scores were calculated by computing the mean score of the items, resulting in a scale ranging $1-5$, and was treated as continuous in analyses. The support subscales were highly correlated, as baseline correlation was $\rho 0.85, p<.01$.

\section{Confounders}

Since a vast number of studies have established that both older age and being female are associated with increased sleep impairments and increased pain sensitivity (Finan et al., 2013), age and sex were incorporated as covariates in all analyses. Skill level was included as a confounder since high levels of WLC are found in workers in executive and managerial positions, and prevalence rates of WLC are higher in employees with a higher education (Hammig et al., 2009).

Skill levels were determined by level of education or relevant work experience, as specified in STYRK classifications, which is a Norwegian adaptation of the International Standard for Classification of Education (ISCED- 
Fig. 1 Conceptual halflongitudinal mediation model of the relationships between WLC, sleep problems, and NPS

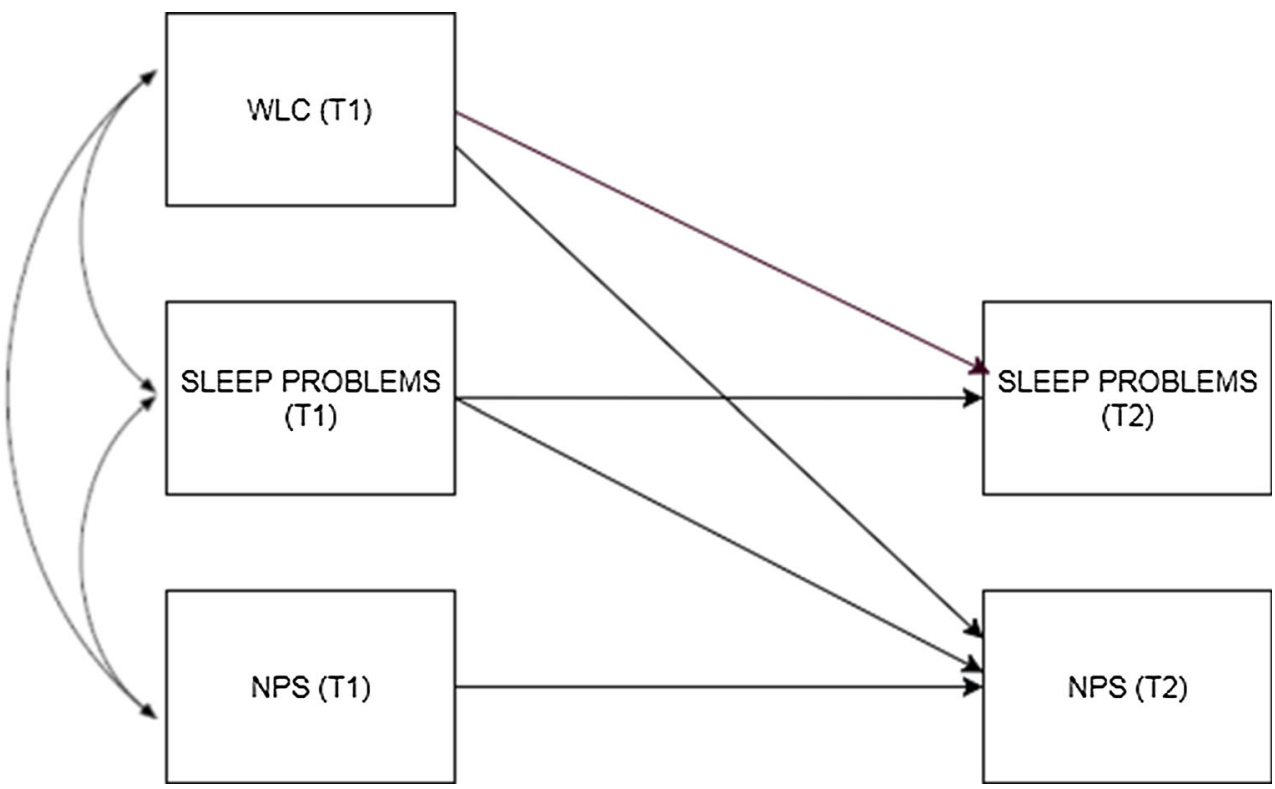

ISCO88). Categories ranged from occupations that require $<10$ years of education to occupations that require $>16$ years of education. Baseline levels of sleep problems and NPS were also included a priori in all regressions.

\section{Statistical analyses}

Analyses were carried out using MPLUS, version 7.4 (Muthén \& Muthén, 1998-2012) and the R package "Mediation" (Tingley et al., 2014).

Prior to mediation analyses, cross-sectional and prospective regressions were conducted to establish the relationships between WLC (predictor), sleep (mediator), and NPS (outcome). Linear regressions were conducted to estimate relationships between WLC and sleep, and Poisson regressions were conducted to estimate relationships between WLC and NPS, and sleep and NPS. These regressions were adjusted for age, sex, and skill level. The prospective regressions were additionally adjusted for the outcome at $\mathrm{T} 1$.

Mediation models reflect how one variable, the exposure, may affect another, the outcome, by introducing a potential intervening variable, a mediator, which is located temporally between exposure and outcome (Hayes, 2013). Ideally, mediation should be studied with three time points to establish the sequential order of exposure, mediator, and outcome. However, for two-wave studies a half-longitudinal model has been proposed by Cole and Maxwell (Cole \& Maxwell, 2003), in which the $\mathrm{T} 1$ exposure predicts the $\mathrm{T} 2$ mediator and the $\mathrm{T} 1$ mediator predicts the $\mathrm{T} 2$ outcome (see Fig. 1). Estimating and testing the mediation effect in such a model involves calculating the product of effect estimates, a method that is not valid with nonlinear regressions (Lee et al., 2016). Since NPS was a count variable, and the effects on NPS were established using Poisson regressions, we employed the half-longitudinal model only for testing the order of effects, i.e. to determine the plausibility of WLC preceding sleep problems and sleep problems preceding pain problems.

The statistical test of mediation was conducted with the mediator (sleep problems) measured at baseline. Since the follow-up period was 2 years, measuring WLC and sleep problems cross-sectionally should reflect reality most accurately if work has a short-term effect on sleep.

Figure 1 illustrates the half-longitudinal models used to test temporal effects. This model included sleep problems at baseline as well as follow-up. Figure 2 illustrates the mediation model where sleep problems at baseline was included. Lastly, Fig. 3 shows the moderated mediation model. In this model as well, sleep at baseline only was included, as the focus here was on whether the association of WLC with impaired sleep is modified by the level of experienced support during the same period.

Since NPS is a count variable, mediation was tested using causal mediation analyses based on counterfactuals (Muthen et al., 2016). Since these analyses are based on comparison of two groups, each level of WLC was compared with the reference level of WLC, which was set to 1 . In counterfactual analyses moderated mediation is tested on both the direct and indirect effect.

Age, sex, skill level, as well as baseline levels of corresponding sleep problems and NPS were entered as covariates in all models.

Bootstrapping methods were performed. Bootstrapping refers to a resampling procedure in which a large number of new datasets are resampled from the existing sample in order to estimate parameters with minimal error. Biascorrected bootstrap confidence interval levels (BCa CI) 
Fig. 2 Conceptual mediation model of the indirect relationships between WLC, sleep problems, and NPS
Fig. 3 Conceptual model of the moderated mediation effect of support on the indirect relationships between WLC, sleep problems, and NPS
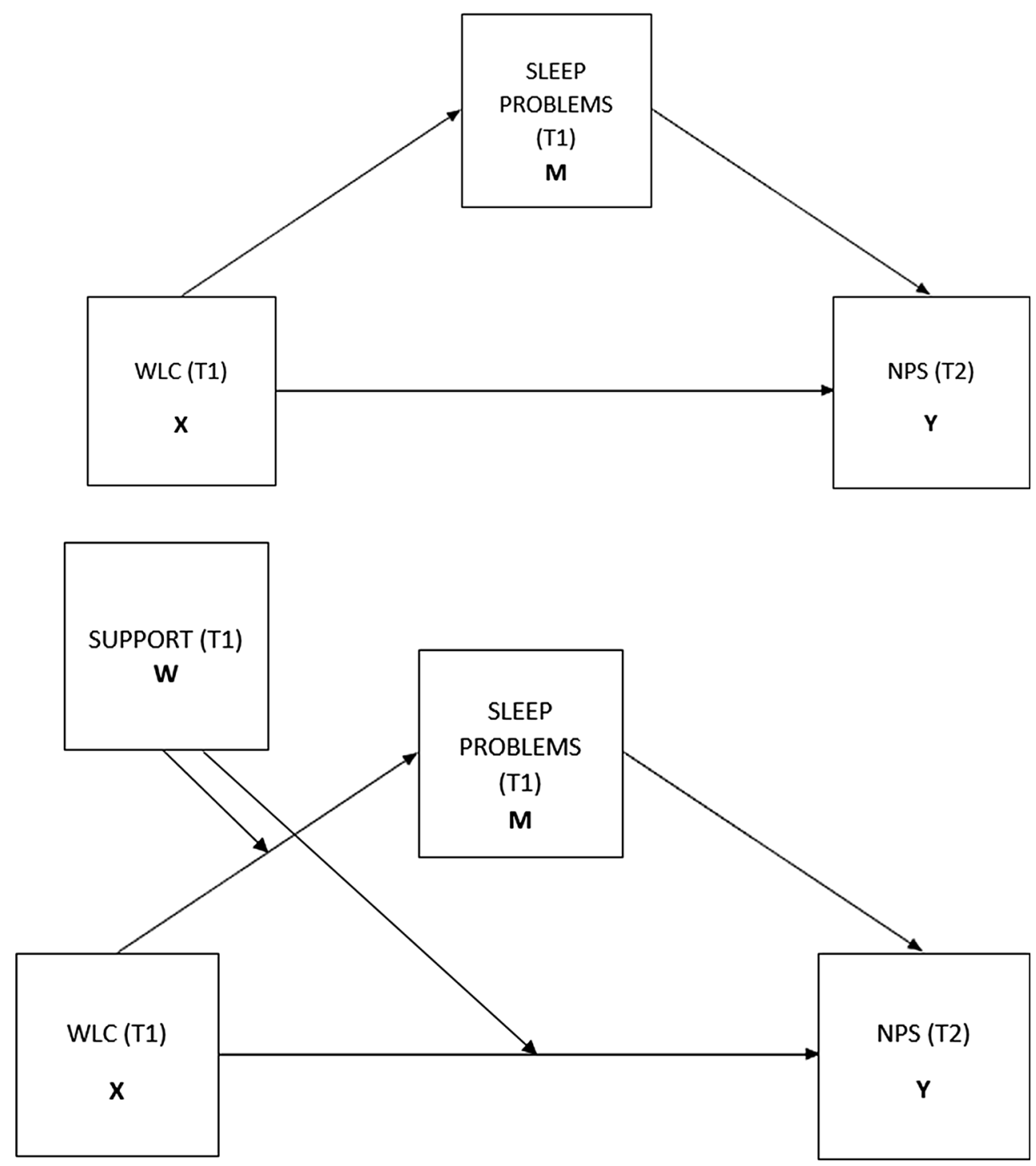

between variables are reflected Table 5, which can be found in the online appendix.

Prior to main analyses, cross-sectional and prospective direct effects were tested. The direct effects of WLC on NPS (IRR $=1.04,95 \%$ CI 1.01-1.06, $p=.02)$, and of sleep problems on NPS were statistically significant $($ IRR $=1.07,95 \%$ CI $1.04-1.09, p<.01)$. After this, in line with the recommendation of Baron and Kenny (Baron \& Kenny, 1986), mutually adjusted direct effects were tested. The direct effect of WLC on NPS was no longer statistically significant $(\mathrm{IRR}=1.02,95 \%$ CI $0.99-1.05$, $p=.18$ ) after adjusting for sleep problems, consistent with a hypothesis of mediation (Baron \& Kenny, 1986).

An overview of these cross-sectional relations, as well as prospective direct effects, including bootstrapped confidence intervals, can be found in Table 2 . 
Table 2 Results from cross-sectional and prospective regressions of outcome (number of pain sites, NPS) on exposure (work-private life conflict, WLC) and mediator (sleep problems, SLEEP), and of mediator on exposure $(\mathrm{N}=4681)$

\begin{tabular}{|c|c|c|c|c|c|}
\hline & & Estimate & IRR & $95 \% \mathrm{CI}, \mathrm{IRR}$ & $p$ \\
\hline \multicolumn{6}{|l|}{ Cross-sectional models } \\
\hline \multicolumn{6}{|l|}{ Univariable regressions } \\
\hline Exposure $\rightarrow$ outcome & $\mathrm{WLC} \rightarrow \mathrm{NPS}$ & 0.14 & 1.15 & [1.12-1.18] & $<0.01$ \\
\hline Mediator $\rightarrow$ outcome & SLEEP $\rightarrow$ NPS & 0.21 & 1.23 & {$[1.21-1.26]$} & $<0.01$ \\
\hline Exposure $\rightarrow$ mediator & WLC $\rightarrow$ SLEEP & 0.230 & - & {$[0.26-0.336]$} & $<0.01$ \\
\hline \multicolumn{6}{|l|}{ Multivariable regression } \\
\hline \multirow[t]{2}{*}{ Exposure + mediator $\rightarrow$ outcome } & $\mathrm{WLC} \rightarrow \mathrm{NPS}$ & 0.08 & 1.08 & [1.05-1.12] & $<0.01$ \\
\hline & SLEEP $\rightarrow$ NPS & 0.20 & 1.22 & [1.19-1.24] & $<0.01$ \\
\hline \multicolumn{6}{|l|}{ Prospective models } \\
\hline \multicolumn{6}{|l|}{ Univariable regressions } \\
\hline Exposure $\rightarrow$ outcome & WLC $\rightarrow$ NPS & 0.04 & 1.04 & {$[1.01-1.01]$} & 0.02 \\
\hline Mediator $\rightarrow$ outcome & SLEEP $\rightarrow$ NPS & 0.06 & 1.07 & [1.04-1.09] & $<0.01$ \\
\hline Exposure $\rightarrow$ mediator & WLC $\rightarrow$ SLEEP & 0.04 & - & {$[0.01-0.07]$} & $<\mathbf{0 . 0 2}$ \\
\hline \multicolumn{6}{|l|}{ Multivariable regression } \\
\hline \multirow[t]{2}{*}{ Exposure + mediator $\rightarrow$ outcome } & WLC $\rightarrow$ NPS & 0.02 & 1.02 & [0.99-1.05] & 0.18 \\
\hline & SLEEP $\rightarrow$ NPS & 0.06 & 1.06 & [1.04-1.09] & $<\mathbf{0 . 0 1}$ \\
\hline
\end{tabular}

All regressions were adjusted for age, sex, and skill level, and prospective regressions adjusted for the baseline level of the dependent variable $I R R$ incident rate ratio

$p<0.05$ marked in bold font

\section{Half-longitudinal mediation analysis}

Temporal relations of direct effects were further examined through the half-longitudinal model, which revealed statistically significant results for all prospective relations tested; the direct effect of WLC at baseline on sleep problems at follow up $(B=0.04,95 \%$ CI $0.01-0.07$, $p=.02)$, and the effect of sleep problems at baseline on NPS at follow-up ( $B=0.06,95 \%$ CI $0.04-0.08, p<.01)$.

\section{Mediation analysis}

As mentioned before, each level of WLC was compared with the reference level of WLC, which was set to 1 . Total effects, direct effects, indirect effects, and proportions mediated for each level of WLC can be found in Table 3.

Significant indirect effects of WLC on NPS through sleep problems were established for each level of WLC, implying that employees experiencing any level of worklife conflict above a WLC score of one also reported higher numbers of pain sites, and this was mediated by higher levels of sleep problems. Direct effects of WLC on NPS were not significant for any level of WLC.

The proportion of the effect of WLC on NPS that is mediated by sleep problems varies slightly between the differing levels measured, from $43 \%$ at level two (low level of WLC) to $42.2 \%$ at level five (high level of WLC), and is significant at all levels.

\section{Moderated mediation analysis}

Prior to main moderated mediation analysis, the moderating role of support on the relationship between WLC at T1 and NPS at T2, not mediated by sleep, was tested $(B=0.03,95 \%$ CI $0.02-0.04, p<.01)$, indicating that the direct effect of WLC on NPS, tested outside the mediation models, was partially dependent on levels of support.

Pairwise t-tests were performed to compare the average direct and indirect effects between all the different pairs of levels of support (see Table 5 online appendix) (Tingley et al., 2014). Moderated mediation analyses confirmed the same pattern as the mediation analyses for all levels of support. That is, regardless of the level of support a statistically significant mediation effect was observed, but no significant direct effects. Although the pattern of associations suggested a trend with the magnitude of the indirect effect decreasing for higher levels of support, no statistically significant differences between indirect effects across levels of support were observed. Hence, there was not evidence that support moderates the effect of WLC on NPS through sleep problems (Table 4). 
Table 3 Mediation of the association between work-private life conflict (WLC) and number of pain sites (NPS) through sleep problems: Direct effects (WLC $\rightarrow$ NPS), indirect effects (WLC $\rightarrow$ sleep $\rightarrow$ NPS), total effects (direct + indirect effect), and the proportion of the total effect that is mediated by sleep problems $(\mathrm{N}=4681)$

\begin{tabular}{|c|c|c|c|c|}
\hline & & Estimate & $95 \% \mathrm{CI}$ & $\mathrm{p}$ \\
\hline WLC level 1 & Comparison level & - & - & - \\
\hline \multirow[t]{4}{*}{ WLC level 2} & Total effect & 0.07 & {$[0.02-0.11]$} & $<0.01$ \\
\hline & Indirect effect & 0.03 & {$[0.02-0.04]$} & $<0.01$ \\
\hline & Direct effect & 0.04 & {$[-0.01-0.08]$} & 0.11 \\
\hline & Proportion mediated & 0.43 & {$[0.23-1.42]$} & $<0.01$ \\
\hline \multirow[t]{4}{*}{ WLC level 3} & Total effect & 0.13 & {$[0.04-0.23]$} & $<\mathbf{0 . 0 1}$ \\
\hline & Indirect effect & 0.06 & {$[0.04-0.08]$} & $<0.01$ \\
\hline & Direct effect & 0.08 & {$[-0.02-0.17]$} & 0.11 \\
\hline & Proportion mediated & 0.43 & {$[0.22-1.34]$} & $<\mathbf{0 . 0}$ \\
\hline \multirow[t]{4}{*}{ WLC level 4} & Total effect & 0.20 & {$[0.06-0.34]$} & 0.01 \\
\hline & Indirect effect & 0.08 & {$[0.05-0.12]$} & $<\mathbf{0 . 0 1}$ \\
\hline & Direct effect & 0.11 & {$[-0.03-0.26]$} & 0.11 \\
\hline & Proportion mediated & 0.43 & {$[0.21-1.35]$} & 0.01 \\
\hline \multirow[t]{4}{*}{ WLC level 5} & Total effect & 0.26 & {$[0.06-0.47]$} & 0.01 \\
\hline & Indirect effect & 0.11 & {$[0.05-0.17]$} & $<\mathbf{0 . 0 1}$ \\
\hline & Direct effect & 0.15 & {$[-0.04-0.35]$} & 0.12 \\
\hline & Proportion mediated & 0.42 & [0.18-1.38] & 0.01 \\
\hline
\end{tabular}

Mediation analyses with counterfactuals require comparison of two groups. Hence, each level of WLC was compared with the reference level of WLC, which was set to 1

$p<0.05$ marked in bold font

Confidence intervals based on nonparametric bootstrapping, 5000 draws

\section{Discussion}

The current study established that work-private life conflict (WLC) predicted the number of pain sites (NPS) (H1), sleep problems predicted NPS (H2), and sleep mediated the effects of WLC on NPS (H3). Lastly, the study investigated the potential buffering effects of work-related social support on the indirect relationship of WLC on NPS (H4), but found no statistically significant moderated mediation effects of support.

The present study suggested that there are time-lagged effects over a 2-year period, of WLC on NPS, as suggested in our first hypothesis. Furthermore, prospective effects of sleep problems on NPS were established, confirming the second hypothesis. Previous research supports these finding, as both WLC and sleep problems have been found to have negative effects on employee health (Doi et al., 2003; Grant-Vallone \& Donaldson, 2001). Frone (2003) in his review on the effects of work-life conflict and work-life balance, concluded that work-life conflict affects psychological distress and mood disorders, self-reported poor physical health, and substance abuse in employees. Sleep problems have been associated with increased levels of sick leave, as well as with poor physical- and psychological health (Doi et al., 2003). Since these modifiable factors have such a visible impact on employee health, and therefore come with a significant societal cost, workplace interventions may wish to target WLC and sleep, which may lower levels of reported pain as well as improving general employee health over time.

Furthermore, the suggested prospective relationships of WLC on NPS are mediated by sleep problems, as suggested by the third hypothesis. Hence, as a result of conflict between their work- and private life, employees may experience sleep problems, which may contribute to multisite musculoskeletal pain complaints and an increase in number of pain sites.

Finally, support did not significantly moderate the indirect effect of WLC on NPS, i.e. levels of support at work from both supervisor as well as coworkers have not been found to buffer the negative effects of WLC on sleep and number of pain sites, which means hypothesis four is not supported by the findings. However, previous studies suggest support may affect sleep problems individually (Akerstedt et al., 2002). Although support may not be sufficient to substantially alleviate the aversive effects of work-life conflict on sleep and pain, it should be noted that it may still play a role in the work-pain relationship. A supportive supervisor who shows understanding of private life demands may for example be forthcoming in solutions 
Table 4 Moderated mediation: total, direct, and indirect effects of work-private life conflict on NPS through sleep problems for different levels of perceived support from superiors and coworkers

\begin{tabular}{|c|c|c|c|c|c|c|}
\hline \multirow[t]{2}{*}{ WLC comparison } & \multirow[t]{2}{*}{ Effect } & \multicolumn{5}{|c|}{ Level of support } \\
\hline & & 1 & 2 & 3 & 4 & 5 \\
\hline \multirow[t]{2}{*}{2 versus 1} & Direct & -0.05 & -0.02 & 0.00 & 0.03 & 0.05 \\
\hline & Indirect & 0.04 & 0.04 & 0.03 & 0.03 & 0.02 \\
\hline \multirow[t]{2}{*}{3 versus 1} & Direct & -0.106 & -0.04 & 0.01 & 0.05 & 0.10 \\
\hline & Indirect & 0.08 & 0.07 & 0.06 & 0.05 & 0.05 \\
\hline \multirow[t]{2}{*}{4 versus 1} & Direct & -0.14 & -0.06 & 0.01 & 0.08 & 0.15 \\
\hline & Indirect & 0.12 & 0.11 & 0.10 & 0.08 & 0.07 \\
\hline \multirow[t]{2}{*}{5 versus 1} & Direct & -0.19 & -0.08 & 0.02 & 0.11 & 0.20 \\
\hline & Indirect & 0.16 & 0.15 & 0.13 & 0.11 & 0.10 \\
\hline
\end{tabular}

None of the effects of support on the indirect relationship between WLC and NPS established were statistically significant at $p<0.05$

when, often unplanned, private life commitments tip the balance. Support may relieve some of the worries employees may experience when work-related expectations are not met due to limited resources, for example, when an employee needs to leave early because of such home-life related engagements.

The present results provide insight into possible mechanisms through which psychological and social work factors may affect pain. It may be that the conflict between demands from work and home affect sleep because of their dual claim on common resources, e.g. time, and findings may be used in the developments of work interventions., since WLC, sleep, and support are all modifiable factors.

\section{Limitations}

Both support and WLC items come from the QPSNordic, a reliable and validated questionnaire (Dallner et al., 2000). Items comprising the WLC scale included in the present study do not constitute a scale in the QPSNordic, rather they are two single items reflecting work-private life interactions. The support scale in the QPSNordic comprises more than the coworker- and superior support items included in the present study. The authors chose to focus primarily on work-based support. Sleep items do not compromise a scale, but have been measured together in other studies (Vleeshouwers et al., 2016). What is more, all variables reflecting measures included in the present study show high correlations between items.

NPS appears to be stable across time, with little variance between baseline and follow-up measures. It is important to note that this does not mean no change on the individual level has occurred between the two time points. While the average change in NPS is moderate, individual levels of NPS may have fluctuated more than the sample level change reflects. High stability combined with baseline adjustment may affect analyses by making effects more difficult to detect.

Only a small number of participants experience high levels of conflict between work- and private life and/or low levels of support. These groups may have simply been too small to establish any effect if present. Since counterfactual mediation analysis relies on comparing groups, the absence of a moderated mediation effect of support could be due to a lack of statistical power. The fact that so few employees experience these 'extremes' in these work factors may be due to Norway's relatively stable and modern workforce (Abendroth \& den Dulk, 2011; Crompton \& Lyonette, 2006). Moreover, Norwegian work culture is characterized by equality and flexibility, with a dual-earner model with both mother and father in paid work, and with extended childcare- and annual leave arrangements, and often with flexible working arrangements available (Kvande, 2009). When compared to other cultures, such as for instance the US, where gender roles in employment appear more visible and the man is often the breadwinner, and where rules and regulations are less forthcoming (Allen et al., 2015), clear differences in levels of work-private life conflict are observed. In Norway, workers in full-time employment have an average of $15.5 \mathrm{~h}$ daily to spend on leisure and personal care, while workers in the US have $1 \mathrm{~h}$ less (OECD, 2015).

While the present study has shed some light on possible mechanism explaining relationships between WLC, and pain, more research is needed in order to improve our understanding of underlying mechanisms. Because of the 2 year time frame, it is possible that effects are transmitted through alternative causal chains, or that there are additional or alternative causes (Shrout \& Bolger, 2002). Other factors are likely to play a part in the multifaceted relationship between work, sleep, and multisite pain. Depression caused by work-factors could affect sleep (Bonde, 2008), which in turn affects pain. Similarly, one could 
argue that appraisal and coping, exercise, and biomechanical exposures at work, may influence these complex relationships. The purpose of the current study was to investigate the role of sleep in mediating the relationship between work-private life conflict and pain. While we did have access to some information about mental health, we chose not to include this in the present analyses. While individuals experiencing mental distress could be more vulnerable to effects of work-private life conflict on sleep and/or pain, it may also be that the experience of WLC affects psychological status, which in turn may affect sleep and/or pain. Confounding is another possibility, if for instance distress affects both WLC, sleep, and pain. We chose not to include mental distress as a control variable since it would be unclear whether any impact on results would be due to effect modification, mediation, or confounding. Moreover, baseline adjustment for NPS should attenuate confounding if distress influenced WLC and NPS similarly across time. Nevertheless, the possible influence of distress should be kept in mind when interpreting results. Future studies may wish to address distress and other potential mediators and moderators that may be a part of the work-pain mechanism.

Organizations partaking in the study received feedback on all psychological and social work factors studied in the form of reports and presentations after each round of data collection. While it is possible that some companies chose to change some of their work practices based on feedback received after $\mathrm{T} 1$, no significant differences in WLC between $\mathrm{T} 1$ and $\mathrm{T} 2$ were found in post hoc analyses in the overall sample.

This study indicated that WLC affected NPS, partly through influencing sleep problems. Social support at work did not significantly moderate this relationship, but may still affect sleep problems and NPS. Although much is still unclear about causal mechanisms, workplace interventions have been shown to improve employee control, workfamily conflict, and sleep (Olson et al., 2015). Findings may be used towards the construction of workplace intervention initiatives that alleviate WLC and improve supportive behaviors in the workplace. One central implication for organizational practitioners is that in cases where work-private-life conflict cannot be avoided organizational support may be particularly important in order to alleviate undesirable consequences. When the bidirectional relationship between work and private life results in positive spillover, sleep may be improved (Williams et al., 2006). Workplace interventions aimed at decreasing overtime work and spillover between employees' private life- and work life, and to improve employees' control over work and home balance, as well as improving communications and support mechanisms at work could significantly improve employee health and well-being, which in turn could benefit productivity and decrease sickness absence.

Acknowledgements The authors wish to thank Bjørn Lau, Anne Lene Andersen, Shahrooz Elka, Elisabeth Petersen, Margrethe Schøning, and Jan S. Emberland for their help in survey administration, as well as all participating companies.

Funding This work was funded by the Norwegian Research Council (Grant Number: 185209). The funders had no role in study design, data collection and analysis, decision to publish, or preparation of the manuscript.

\section{Compliance with ethical standards}

Conflict of interest Jolien Vleeshouwers, Stein Knardahl and Jan Olav Christensen declare that they have no conflict of interest.

Human and animal rights and Informed consent All procedures performed in studies involving human participants were in accordance with the ethical standards of the institutional and/or national research committee and with the 1964 Helsinki declaration and its later amendments or comparable ethical standards. Informed consent was obtained from all individual participants included in the study.

Open Access This article is distributed under the terms of the Creative Commons Attribution 4.0 International License (http:// creativecommons.org/licenses/by/4.0/), which permits unrestricted use, distribution, and reproduction in any medium, provided you give appropriate credit to the original author(s) and the source, provide a link to the Creative Commons license, and indicate if changes were made.

\section{References}

Abendroth, A. K., \& den Dulk, L. (2011). Support for the work-life balance in Europe: The impact of state, workplace and family support on work-life balance satisfaction. Work, Employment \& Society, 25, 234-256. https://doi.org/10.1177/ 0950017011398892

Aili, K., Nyman, T., Svartengren, M., \& Hillert, L. (2015). Sleep as a predictive factor for the onset and resolution of multi-site pain: A 5-year prospective study. European Journal of Pain, 19, 341-349. https://doi.org/10.1002/ejp.552

Akerstedt, T., Knutsson, A., Westerholm, P., Theorell, T., Alfredsson, L., \& Kecklund, G. (2002). Sleep disturbances, work stress and work hours: A cross-sectional study. Journal of Psychosomatic Research, 53, 741-748.

Allen, T. D., French, K. A., Dumani, S., \& Shockley, K. M. (2015). Meta-analysis of work-family conflict mean differences: Does national context matter? Journal of Vocational Behavior, 90, 90-100. https://doi.org/10.1016/j.jvb.2015.07.006

Alsaadi, S. M., McAuley, J. H., Hush, J. M., Lo, S., Bartlett, D. J., Grunstein, R. R., et al. (2014). The bidirectional relationship between pain intensity and sleep disturbance/quality in patients with low back pain. Clinical Journal of Pain, 30, 755-765. https://doi.org/10.1097/AJP.0000000000000055

Barnes, C. M., Wagner, D. T., \& Ghumman, S. (2012). Borrowing from sleep to pay work and family: Expanding time-based conflict to the broader nonwork domain. Personnel Psychology, 65, 789-819. https://doi.org/10.1111/peps. 12002 
Baron, R. M., \& Kenny, D. A. (1986). The moderator mediator variable distinction in social psychological-research-Conceptual, strategic, and statistical considerations. Journal of Personality and Social Psychology, 51, 1173-1182. https://doi.org/10. 1037/0022-3514.51.6.1173

Berntsson, L., Lundberg, U., \& Krantz, G. (2006). Gender differences in work-home interplay and symptom perception among Swedish white-collar employees. Journal of Epidemiology and Community Health, 60, 1070-1076. https://doi.org/10.1136/jech. 2005.042192

Bonde, J. P. E. (2008). Psychosocial factors at work and risk of depression: A systematic review of the epidemiological evidence. Occupational and Environmental Medicine, 65, 438-445. https://doi.org/10.1136/oem.2007.038430

Bongers, P. M., Dewinter, C. R., Kompier, M. A. J., \& Hildebrandt, V. H. (1993). Psychosocial factors at work and musculoskeletal disease. Scandinavian Journal of Work, Environment \& Health, 19, 297-312.

Christensen, J. O., \& Knardahl, S. (2010). Work and neck pain: A prospective study of psychological, social, and mechanical risk factors. Pain, 151, 162-173. https://doi.org/10.1016/j.pain.2010. 07.001

Christensen, J. O., \& Knardahl, S. (2012). Work and back pain: A prospective study of psychological, social and mechanical predictors of back pain severity. European Journal of Pain (London, England), 16(6), 921-933. https://doi.org/10.1002/j. 1532-2149.2011.00091.x

Coggon, D., Ntani, G., Palmer, K. T., Felli, V. E., Harari, R., Barrero, L. H., et al. (2013). Patterns of multisite pain and associations with risk factors. Pain, 154, 1769-1777. https://doi.org/10.1016/ j.pain.2013.05.039

Cole, D. A., \& Maxwell, S. E. (2003). Testing mediational models with longitudinal data: Questions and tips in the use of structural equation modeling. Journal of Abnormal Psychology, 112, 558-577. https://doi.org/10.1037/0021-843x.112.4.558

Crain, T. L., Hammer, L. B., Bodner, T., Kossek, E. E., Moen, P., Lilienthal, R., et al. (2014). work-family conflict, familysupportive supervisor behaviors (FSSB), and sleep outcomes. Journal of Occupational Health Psychology, 19, 155-167. https://doi.org/10.1037/a0036010

Crompton, R., \& Lyonette, C. (2006). Work-life 'balance' in Europe. Acta Sociologica, 49, 379-393. https://doi.org/10.1177/ 0001699306071680

Dallner, M., Elo, A. L., Gamberale, F., Hottinen, V., Knardahl, S., Lindström, K., et al. (2000). Validation of the General Nordic Questionnaire (QPSNordic) for psychological and social factors at work (p. 12). Nord: Nordic Council of Ministers.

Davis, K. G., \& Heaney, C. A. (2000). The relationship between psychosocial work characteristics and low back pain: Underlying methodological issues. Clinical Biomechanics (Bristol, Avon), 15(6), 389-406.

Dean, L. E., Arnold, L., Crofford, L., Bennett, R., Goldenberg, D., Fitzcharles, M.-A., et al. (2017). The impact of moving from a widespread to multi-site pain definition on other fibromyalgia symptoms. Arthritis Care \& Research. https://doi.org/10.1002/ acr.23214

Demerouti, E., Derks, D., ten Brummelhuis, L. L., \& Bakker, A. B. (2014). New ways of working: Impact on working conditions, work-family balance, and well-being. In C. Korunka \& P. Hoonakker (Eds.), The impact of ICT on quality of working life (pp. 123-141). Dordrecht: Springer.

Doi, Y., Minowa, M., \& Tango, T. (2003). Impact and correlates of poor sleep quality in Japanese white-collar employees. Sleep, 26, 467-471. https://doi.org/10.1093/sleep/26.4.467

Eriksen, W., Bruusgaard, D., \& Knardahl, S. (2004). Work factors as predictors of intense or disabling low back pain; A prospective study of nurses' aides. Occupational and Environmental Medicine, 61, 398-404.

Finan, P. H., Goodin, B. R., \& Smith, M. T. (2013). The association of sleep and pain: An update and a path forward. Journal of Pain, 14, 1539-1552. https://doi.org/10.1016/j.jpain.2013.08. 007

Frone, M. R. (2003). Work-family balance. In J. C. Quick \& L. E. Tetric (Eds.), Handbook of occupational health psychology (pp. 143-162). Washington, DC: American Psychological Association.

Ganster, D. C., Fusilier, M. R., \& Mayes, B. T. (1986). Role of social support in the experience of stress at work. Journal of Applied Psychology, 71, 102-110. https://doi.org/10.1037/0021-9010.71. 1.102

Grant-Vallone, E. J., \& Donaldson, S. I. (2001). Consequences of work-family conflict on employee well-being over time. Work and Stress, 15, 214-226. https://doi.org/10.1080/ 02678370110066544

Haack, M., Sanchez, E., \& Mullington, J. M. (2007). Elevated inflammatory markers in response to prolonged sleep restriction are associated with increased pain experience in healthy volunteers. Sleep, 30, 1145-1152.

Hammig, O., \& Bauer, G. F. (2014). Work, work-life conflict and health in an industrial work environment. Occupational Medicine (Oxford, England), 64(1), 34-38. https://doi.org/10. 1093/occmed/kqt127

Hammig, O., Gutzwiller, F., \& Bauer, G. (2009). Work-life conflict and associations with work- and nonwork-related factors and with physical and mental health outcomes: A nationally representative cross-sectional study in Switzerland. BMC Public Health, 9, 435. https://doi.org/10.1186/1471-2458-9-435

Harvey, A. G., Stinson, K., Whitaker, K. L., Moskovitz, D., \& Virk, H. (2008). The subjective meaning of sleep quality: A comparison of individuals with and without insomnia. Sleep, 31, 383-393.

Haukka, E., Kaila-Kangas, L., Ojajarvi, A., Miranda, H., Karppinen, J., Viikari-Juntura, E., et al. (2013). Pain in multiple sites and sickness absence trajectories: A prospective study among Finns. Pain, 154, 306-312. https://doi.org/10.1016/j.pain.2012.11.003

Hayes, A. F. (2013). Introduction to mediation, moderation, and conditional process analysis: A regression-based approach. New York: Guilford Press.

Hayes, A. F., \& Scharkow, M. (2013). The relative trustworthiness of inferential tests of the indirect effect in statistical mediation analysis: Does method really matter? Psychological Science, 24, 1918-1927. https://doi.org/10.1177/0956797613480187

Hobfoll, S. E., Freedy, J., Lane, C., \& Geller, P. (1990). Conservation of social resources-Social support resource theory. Journal of Social and Personal Relationships, 7, 465-478. https://doi.org/ 10.1177/0265407590074004

Hoogendoorn, W. E., van Poppel, M. N. M., Bongers, P. M., Koes, B. W., \& Bouter, L. M. (2000). Systematic review of psychosocial factors at work and private life as risk factors for back pain. Spine, 25, 2114-2125. https://doi.org/10.1097/00007632200008150-00017

Jacobsen, H. B., Reme, S. E., Sembajwe, G., Hopcia, K., Stoddard, A. M., Kenwood, C., et al. (2014). Work-family conflict, psychological distress, and sleep deficiency among patient care workers. Workplace Health \& Safety, 62, 282-291. https://doi. org/10.3928/21650799-20140617-04

Kamaleri, Y., Natvig, B., Ihlebaek, C. M., Benth, J. S., \& Bruusgaard, D. (2008a). Number of pain sites is associated with demographic, lifestyle, and health-related factors in the general population. European Journal of Pain, 12, 742-748. https:// doi.org/10.1016/j.ejpain.2007.11.005 
Kamaleri, Y., Natvig, B., Ihlebaek, C. M., \& Bruusgaard, D. (2008b). Localized or widespread musculoskeletal pain: Does it matter? Pain, 138, 41-46. https://doi.org/10.1016/j.pain.2007.11.002

Kamaleri, Y., Natvig, B., Ihlebaek, C. M., \& Bruusgaard, D. (2009). Does the number of musculoskeletal pain sites predict work disability? A 14-year prospective study. European Journal of Pain, 13, 426-430. https://doi.org/10.1016/j.ejpain.2008.05.009

Kim, S. S., Okechukwu, C. A., Buxton, O. M., Dennerlein, J. T., Boden, L. I., Hashimoto, D. M., et al. (2013). Association between work-family conflict and musculoskeletal pain among hospital patient care workers. American Journal of Industrial Medicine, 56, 488-495. https://doi.org/10.1002/ajim.22120

Kossek, E. E., Pichler, S., Bodner, T., \& Hammer, L. B. (2011). Workplace social support and work-family conflict: A metaanalysis clarifying the influence of general and work-familyspecific supervisor and organizational support. Personnel Psychology, 64, 289-313. https://doi.org/10.1111/j.1744-6570.2011. 01211.x

Kvande, E. E. (2009). Work-life balance for fathers in globalized knowledge work. Some insights from the Norwegian context. Gender, Work and Organization, 16, 58-72.

Lallukka, T., Rahkonen, O., Lahelma, E., \& Arber, S. (2010). Sleep complaints in middle-aged women and men: The contribution of working conditions and work-family conflicts. Journal of Sleep Research, 19, 466-477. https://doi.org/10.1111/j.1365-2869. 2010.00821.x

Lee, H., Hubscher, M., \& McAuley, J. H. (2016). The implications of using binary outcomes in mediation analysis. Journal of Pain, 17, 1045-1046. https://doi.org/10.1016/j.jpain.2016.03.007

Lindström, K. (2000). User's guide for the QPSNordic: General Nordic questionnaire for psychological and social factors at work. Copenhagen: Nordic Council of Ministers.

Little, T. D. (2013). Longitudinal structural equation modeling. New York: The Guilford Press.

Magnusson Hanson, L. L., Chungkham, H. S., Åkerstedt, T., \& Westerlund, H. (2014). The role of sleep disturbances in the longitudinal relationship between psychosocial working conditions, measured by work demands and support, and depression. Sleep, 37, 1977-1985. https://doi.org/10.5665/sleep.4254

Matre, D., Hu, L., Viken, L. A., Hjelle, I. B., Wigemyr, M., Knardahl, S., et al. (2015). Experimental sleep restriction facilitates pain and electrically induced cortical responses. Sleep, 38, 1607-1617. https://doi.org/10.5665/sleep.5058

Miranda, H., Kaila-Kangas, L., Heliovaara, M., Leino-Arjas, P., Haukka, E., Liira, J., et al. (2010). Musculoskeletal pain at multiple sites and its effects on work ability in a general working population. Occupational and Environmental Medicine, 67, 449-455. https://doi.org/10.1136/oem.2009.048249

Moldofsky, H. (2001). Sleep and pain. Sleep Medicine Reviews, 5, 385-396. https://doi.org/10.1053/smrv.2001.0179

Muthén, L. K., \& Muthén, B. O. (1998-2012). Mplus user's guide (7th ed.). Los Angeles, CA: Muthén \& Muthén.

Muthen, B. O., Muthen, L. K., \& Asparouhov, T. (2016). Regression and mediation analysis using Mplus. Los Angeles: Muthen \& Muthen.
Nakata, A., Haratani, T., Takahashi, M., Kawakami, N., Arito, H., Kobayashi, F., et al. (2004). Job stress, social support, and prevalence of insomnia in a population of Japanese daytime workers. Social Science and Medicine, 59, 1719-1730. https:// doi.org/10.1016/j.socscimed.2004.02.002

Neupane, S., Miranda, H., Virtanen, P., Siukola, A., \& Nygard, C. H. (2013). Do physical or psychosocial factors at work predict multi-site musculoskeletal pain? A 4-year follow-up study in an industrial population. International Archives of Occupational and Environmental Health, 86, 581-589. https://doi.org/10.1007/ s00420-012-0792-2

OECD. (2015). How's life? 2015: Measuring well-being. Paris: OECD Publishing.

Olson, R., Crain, T. L., Bodner, T. E., King, R., Hammer, L. B., Klein, L. C., et al. (2015). A workplace intervention improves sleep: Results from the randomized controlled work, family, and health study. Sleep Health, 1, 55-65. https://doi.org/10.1016/j. sleh.2014.11.003

Paananen, M. (2011). Multi-site musculoskeletal pain in adolescence: Occurrence, determinants, and consequences. Oulu: University of Oulu.

Schmidt, C. O., \& Baumeister, S. E. (2007). Simple patterns behind complex spatial pain reporting? Assessing a classification of multisite pain reporting in the general population. Pain, 133, 174-182. https://doi.org/10.1016/j.pain.2007.04.022

Shrout, P. E., \& Bolger, N. (2002). Mediation in experimental and nonexperimental studies: New procedures and recommendations. Psychological Methods, 7, 422-445. https://doi.org/10.1037// 1082-989x.7.4.422

Sivertsen, B., Lallukka, T., Petrie, K. J., Steingrimsdottir, O. A., Stubhaug, A., \& Nielsen, C. S. (2015). Sleep and pain sensitivity in adults. Pain, 156, 1433-1439. https://doi.org/10.1097/j.pain. 0000000000000131

Solidaki, E., Chatzi, L., Bitsios, P., Markatzi, I., Plana, E., Castro, F., et al. (2010). Work related and psychological determinants of multi-site musculoskeletal pain. Scandinavian Journal of Work, Environment \& Health, 36, 54-61.

Tingley, D., Yamamoto, T., Hirose, K., Keele, L., \& Imai, K. (2014). mediation: R package for causal mediation analysis. Journal of Statistical Software, 59, 1-38.

Vleeshouwers, J., Knardahl, S., \& Christensen, J. O. (2016). Effects of psychological and social work factors on self-reported sleep disturbance and difficulties initiating sleep. Sleep, 39, 833-846.

Williams, A., Franche, R. L., Ibrahim, S., \& Mustard, C. A. (2006). Examining the relationship between work-family spillover and sleep quality. Journal of Occupational Health Psychology, 11, 27-37. https://doi.org/10.1037/1076-8998.11.1.27

Wolfe, F., Clauw, D. J., Fitzcharles, M. A., Goldenberg, D. L., Katz, R. S., Mease, P., et al. (2010). The American College of Rheumatology preliminary diagnostic criteria for fibromyalgia and measurement of symptom severity. Arthritis Care \& Research (Hoboken), 62, 600-610. https://doi.org/10.1002/acr.20140

Woolf, A. D., \& Pfleger, B. (2003). Burden of major musculoskeletal conditions. Bulletin of the World Health Organization, 81, 646-656. 